in vivo $35: 119-129(2021)$

doi:10.21873/invivo.12239

\title{
Inhibition of Colony-Stimulating Factor-1 Receptor Enhances the Efficacy of Radiotherapy and Reduces Immune Suppression in Glioblastoma
}

\author{
MUAYAD F. ALMAHARIQ ${ }^{1 *}$, THOMAS J. QUINN ${ }^{1 *}$, PRAVIN KESARWANI ${ }^{1}$, \\ SHIVA KANT ${ }^{1}$, C. RYAN MILLER ${ }^{2}$ and PRAKASH CHINNAIYAN ${ }^{1,3}$ \\ ${ }^{1}$ Department of Radiation Oncology, Beaumont Health, Royal Oak, MI, U.S.A.; \\ ${ }^{2}$ Division of Neuropathology, Department of Pathology, O'Neal Comprehensive Cancer Center, \\ Comprehensive Neuroscience Center, University of Alabama School of Medicine, Birmingham, AL, U.S.A.; \\ ${ }^{3}$ Oakland University William Beaumont School of Medicine, Rochester, MI, U.S.A.
}

\begin{abstract}
Aim: To use inhibition of colony-stimulating factor-1 receptor $(C S F-1 R)$ to target tumor-associated macrophages (TAMs) and improve the efficacy of radiotherapy in glioblastoma (GBM). Materials and Methods: The CSF-1R inhibitor BLZ-945 was used to examine the impact of CSF-1R inhibition on M2 polarization in vitro. Using an orthotopic, immunocompetent GBM model, mice were treated with vehicle, RT, BLZ-945, or RT plus BLZ-945. Results: BLZ-945 reduced M2 polarization in vitro. BLZ-945 alone did not improve median overall survival ( $m O S=29$ days) compared to control mice ( $m O S=27$ days). $R$ T improved survival $(m O S=45$ days; $p=0.02)$, while RT plus BLZ-945 led to the longest survival ( $m O S=$ not reached; $p=0.005)$. Resected tumors had a relatively large population of M2 TAMs in GBM at baseline, which was increased in response to RT. BLZ-945 reduced RTinduced M2 infiltration. Conclusion: Inhibition of CSF-1R improved response to $R T$ in the treatment of GBM and may represent a promising strategy to improve $R T$-induced antitumor immune responses.
\end{abstract}

Glioblastoma (GBM) is the most common and aggressive primary malignant brain tumor, and represents more than half of all gliomas and $\sim 16 \%$ of all primary brain tumors (1).

This article is freely accessible online.

*These Authors contributed equally to this study.

Correspondence to: Prakash Chinnaiyan, Beaumont Health, 3601 West 13 Mile, Royal Oak, MI 48073, U.S.A. Tel: +1 2485512560, Fax: +1 2485510089, e-mail: prakash.chinnaiyan@beaumont.edu

Key Words: Glioblastoma, CSF-1R, M2 macrophages, immunosuppression.
The standard of care for the treatment of GBM is maximal safe resection followed by concurrent chemoradiotherapy (2). Despite significant improvements in surgical technique and increased accuracy of radiotherapy (RT) delivery in the modern era, GBM remains virtually incurable, with a median overall survival (OS) of less than 2 years (1). One potential strategy to achieve clinical gains is to improve the immune response to GBM, which is particularly adept at creating an immunosuppressive tumor microenvironment that both promotes the survival of tumor cells and mediates radiation resistance (3). Our group and others have shown in animal and human studies that there is a rapid pro-inflammatory response within the GBM milieu after RT, followed by an influx of immunosuppressive cells that temper antitumor immunity (4-6).

Unfortunately, early results of combining immune checkpoint inhibitors with RT for the treatment of GBM have been disappointing. CheckMate-498, a phase III study, randomized 550 patients with newly diagnosed GBM to resection followed by concurrent RT and temozolomide or concurrent RT and nivolumab, an antibody to programmed cell death-1 (PD1), and found no difference in OS between the two arms (7). Another phase III trial, CheckMate-143, compared nivolumab to bevacizumab in the treatment of patients with recurrent GBM and also showed no improvement in OS with nivolumab (8). Multiple other checkpoint inhibitors have been tested in phase II trials in patients with GBM with no promising results (9).

One of the leading hypotheses for the lack of clinical benefit of the commonly used immune checkpoint inhibitors in GBM is that investigations thus far may have focused on the wrong target (10). Drugs such as nivolumab, pembrolizumab, and ipilimumab all reduce immune suppression mediated by regulatory T-cells (Tregs) through blockage of either the PD1/programmed cell death ligand-1 
(PD-L1) axis or cytotoxic T-lymphocyte-associated protein 4 (11). Although Tregs play a crucial role in promoting immunosuppression of cytotoxic T-lymphocytes (CTLs) in multiple solid tumors, recent evidence suggests this role is predominantly performed by tumor associated macrophages (TAMs) in GBM $(10,12)$. Macrophages can be polarized toward a pro-inflammatory/antitumor phenotype (M1), or an anti-inflammatory/immune suppressive phenotype (M2) (13). Tumors have been shown to have the capacity for polarizing macrophages toward the M2 phenotype, and the accumulation of these cells, in turn, helps tumors escape immune surveillance (14). M2 TAMs are found at higher percentages in GBM microenvironments compared to other tumor types, suggesting they play a critical role in immune suppression and can influence RT resistance in this tumor entity $(4,10)$. Hence, there is considerable interest in developing therapeutic strategies designed to inhibit M2 TAMs or revert their polarization to enhance antitumor immunity. There are several ongoing phase I/II studies testing novel TAM-targeting agents (15) but none currently exist for clinical practice.

Colony-stimulating factor- 1 receptor (CSF-1R), through its receptor CSF-R1, plays an important role in macrophage development and differentiation. Binding of CSF-1 to CSF-R1 triggers auto-phosphorylation of the receptor on several tyrosine residues, which can activate multiple intracellular pathways, including phosphatidyl inositol 3-kinase, which promotes macrophage maturation and upregulates expression of genes that lead to the M2 macrophage phenotype (16). Hence, there has been considerable interest in investigating the therapeutic potential of CSF-R1 inhibitors in cancer treatment. In fact, there is an ongoing phase $\mathrm{I} / \mathrm{II}$ trial evaluating the safety and efficacy of BLZ-945, a small-molecule inhibitor of CSF$1 \mathrm{R}$, in patients with a variety of advanced solid tumor types (17). However, the trial is not designed to examine the possible potentiation of RT by BLZ-945 in GBM.

Recent pre-clinical evidence suggested that the use of BLZ-945 alone for the treatment GBM may be insufficient to produce significant improvement in survival, but its combination with RT may enhance RT-induced antitumor immunity and lead to long-term improvement in outcomes. In one study, inhibition of CSF-1R with BLZ-945 using a mouse model of glioma reduced M2 polarization within the tumor microenvironment and reduced tumor growth rates (18). A follow-up study, however, showed inhibition of CSF$1 \mathrm{R}$ alone was insufficient for tumor control and growth resumed after an initial period of slow proliferation (19). Another promising report showed the addition of the CSF-1R inhibitor PLX3397 enhanced the efficacy of RT and reduced infiltration of myeloid suppresser cells in an orthotopic and heterotopic mouse model using the human GBM cell line U251 (20). However, the models were employed in athymic $\mathrm{nu} / \mathrm{nu}$ (nude) mice that lack adaptive immunity, so it is difficult to make conclusions about the interplay between radiation-induced immune responses to the tumor and CSF$1 \mathrm{R}$ inhibition. Here, we examined the potential of the CSF1R inhibitor BLZ-945 to increase the efficacy of RT in GBM using an orthotopic, immunocompetent mouse model and determined its influence on the immune landscape.

\section{Materials and Methods}

M2 macrophage polarization. Polarization of bone-marrow derived monocytes toward an $\mathrm{M} 2$ phenotype was performed as previously described (21). Briefly, bone-marrow cells were harvested from the femora of C57BL/6 mice. Cells were cultured in RPMI-1640 media (ThermoFisher Scientific, Grand Island, NY, USA) in the presence of murine GM-CSF ( $20 \mathrm{ng} / \mathrm{ml}$ ) for 5 days. M2 polarization was then performed with the addition of interleukin-4 (IL4) and IL13 cytokines (Peprotech, Inc., Rockey Hill, NJ, USA) at a concentration of $20 \mathrm{ng} / \mathrm{ml}$ for 2 days in the presence or absence of BLZ-945 (MedKoo Biosciences, Morrisville, NC, USA) at a concentration of $1 \mu \mathrm{M}$. Subsequently, we assessed the proportion of M2 macrophages obtained in relation to total macrophage number using fluorescence-activated cell sorting (FACS). The panmacrophage markers CD45, CD11b, and F4/80 (BioLegend, Sand Diego, CA, USA) were used to identify macrophages, and the CD206 marker (BioLegend, Sand Diego, CA, USA) was used to identify M2 macrophages (22). Antibodies were used at the concentration recommended by the manufacturer.

Animal studies. Animal studies were performed under a protocol approved by the Institutional Animal Care and Use Committee of Beaumont Health based on the Animal Research: Reporting of In Vivo Experiments guidelines (protocol AL-19-07). For all studies, immunocompetent C57BL/6 (H-2b, CD45.2), 6- to 8-week-old, female mice, weighing 21-24 g, were used. Cells which were engineered to have the main altered signaling pathways in human GBM tumors, including the retinoblastoma (T), receptor tyrosine kinase/RAS $(R)$, and the phosphatase and tensin homolog (PTEN; $P$ ) pathways, known as TRP cells, were used. Generation of this cell line was described previously (23-25). We initially performed a pilot study to assess the therapeutic potential of combining BLZ-945 and RT. For each mouse, $1 \times 10^{5}$ cells were injected into the right cerebral hemisphere under general anesthesia and stereotactic guidance. The injection site was $1 \mathrm{~mm}$ posterior and $2 \mathrm{~mm}$ lateral to the bregma. Mice were randomized at four mice per group to treatment with vehicle alone (captisol, a commercially available cyclodextrin; Ligand, San Diego, CA, USA), BLZ-945 alone, RT plus vehicle, or RT plus BLZ-945. BLZ-945 was delivered via oral gavage in $20 \%$ captisol at $200 \mathrm{mg} / \mathrm{kg}$ daily for 10 treatments. RT was whole-brain radiotherapy to 18 Gy in 3 daily fractions. Mice were sacrificed on day 10 post RT and brains were harvested for hematoxylin and eosin (H\&E) staining to assess tumor burden. Brains were systematically sectioned at $10 \mu \mathrm{m}$ intervals through the tumor and adjacent normal brain tissue and $\mathrm{H} \& \mathrm{E}$ slides were prepared using standard staining methodology. Tumor burden was quantified as described previously (26). Briefly, slides were imaged, and the images were imported into Definiens Tissue Studio software (Definiens Inc., Cambridge, MA, USA). The software then segmented the images and an iterative learning algorithm was employed to identify normal brain tissue and tumor cells. Tumor volume was then expressed as a percentage of total normal brain tissue. 
Based on the results of this pilot study, we performed a larger survival study. The orthotopic GBM model was repeated exactly as above. Brain magnetic resonance imaging (MRI) was obtained 8 days after tumor implantation to confirm uptake. Images were uploaded to MIM software (MIM Software, Cleveland, OH, USA) and tumors were contoured for volume measurements. Mice were then stratified by tumor size and randomized to treatment with vehicle, BLZ-945 alone, RT plus vehicle, or RT plus BLZ-945. On day 14 post tumor implantation, the RT groups were treated with 18 Gy whole brain RT in 3 daily fractions. For all groups, inhibitor and vehicle treatment were initiated on day 14 post tumor implantation and administered daily, 5 days a week, for a total of 15 doses. Brain MRI was again obtained 2 weeks after initiation of treatment. Mice were assessed and weighed twice weekly and sacrificed if they lost $20 \%$ of their baseline weight, became symptomatic, or reached the pre-specified time point of 14 weeks. Each treatment arm had 11 mice. At the time of sacrifice, mouse brains were harvested, and tumors were extracted for FACS analysis to assess the ratios of $\mathrm{CD} 45^{+} \mathrm{F} 4 / 80^{+} \mathrm{CD} 206^{+} \mathrm{M} 2$ macrophages, $\mathrm{CD} 3{ }^{+} \mathrm{CD} 8{ }^{+} \mathrm{CTLs}$, and $\mathrm{CD} 4{ }^{+} \mathrm{CD} 25^{+}$Tregs in the tumor microenvironment.

Statistical analysis. Student's $t$-test was used to compare tumor size between the treatment groups on MRI and H\&E staining. Values $p<0.05$ were considered statistically significant. Analysis of OS was performed using the Kaplan-Meier method, with the log-rank method (Mantel-Cox) to assess for significance. In order to account for multiple comparisons testing, the Bonferroni correction was utilized. All statistical analyses were completed using R (v3.6.1) and RStudio (v1.2.5019).

\section{Results}

BLZ-945 reduces $M 2$ macrophage polarization. To assess the role of BLZ-945 in M2 macrophage polarization, we cultured bone-marrow derived monocytes in the presence of CSF for 5 days, followed by conditioning with IL4 and IL13 for 2days with and without the CSF-1R inhibitor BLZ-945. Tumors, including gliomas, secrete these cytokines, which promote M2 polarization of TAM in a paracrine fashion in the tumor microenvironment (27). Inhibition of CSF-1R did not change the total number of macrophages $\left(\mathrm{CD} 45^{+} \mathrm{F} 4 / 80^{+}\right)$but significantly reduced M2 macrophage polarization $\left(\mathrm{CD} 45^{+} \mathrm{F} 4 / 80^{+} \mathrm{CD} 206^{+}\right)$. Specifically, macrophages comprised $80.7 \% \pm 3.4 \%$ and $78.5 \% \pm 2.2 \%$ of all immune cells with and without BLZ-945, respectively (Figure $1 ; p=0.62$ ). Polarizing monocytes toward an M2 phenotype resulted in $35.1 \% \pm 2.3 \%$ enrichment of M2 macrophages, which was reduced by $33.3 \% \pm 1.2 \%$ with the addition of BLZ-945 (Figure $1 ; p=0.009$ ).

BLZ-945 potentiates $R T$ and improves survival of mice with $G B M$. To examine the potential of BLZ-945 to enhance the efficacy of RT in the treatment of GBM, we employed an orthotopic murine GBM model. We used TRP GBM cells, which have been shown to recapitulate both the molecular and histopathological features of human GBM (28). Initially, we conducted a pilot study in which tumor cells were injected intracranially and 2 weeks later, treatment was initiated with vehicle, BLZ-945, RT, or RT plus BLZ-945 ( $n=4$ for all groups) (Figure 2A). Mice were sacrificed, and brains were harvested 10 days after initiation of treatment. As shown in Figure 2B, H\&E staining of the harvested brains showed no difference in tumor burden between the control and BLZ-945-treated groups. RT reduced the tumor burden compared to the control and group treated with BLZ945 but the reduction was not statistically significant. Combination of RT and BLZ-945 resulted in a statistically significant reduction in tumor burden compared to both the control and BLZ-945-treated groups.

Based on the promising results of the pilot study, we proceeded with a larger survival experiment with the same four treatment arms ( $\mathrm{n}=11$ for all groups). To ensure the differences observed in OS were not due to different pretreatment tumor sizes, we obtained brain MRI prior to initiation of treatment and stratified mice by tumor size before randomization. There was no difference in tumor size between any of the four groups before treatment (Figure 3A and B). We repeated MRI 2 weeks after treatment initiation. Treatment with BLZ-945 alone did not significantly reduce tumor growth compared to the control arm, but RT alone did. However, RT was significantly more efficacious when BLZ945 was administered concurrently as demonstrated by smaller tumor volumes (Figure 3A and D).

We evaluated survival by Kaplan-Meier survival estimates (Figure 4). Treatment with RT combined with BLZ-945 resulted in significantly improved OS compared to all other groups. Median OS was 27 days, 29 days, and 45 days in the control, BLZ-945, and RT groups. Median OS was not reached in the group treated with RT and BLZ-945 ( $p=0.005$ vs. RT alone). Cox regression survival analysis showed the lowest risk of death for the combination treatment group (Table I), with $70 \%$ (eight mice) achieving long-term survival and reaching the pre-determined study endpoint. Treatment with BLZ-945 alone did not significantly improve survival. RT alone improved survival compared to control and BLZ-945 alone, but with a lower efficacy compared to their combination. To account for multiple comparisons testing, we applied the Bonferroni correction and the benefit of combination treatment with RT and BLZ-945 compared to RT alone remained significant.

BLZ-945 reduces $M 2$ macrophage infiltration in GBM. We performed correlative studies on the tumors from brains harvested at the time of death from each mouse in all four treatment arms and examined the presence of total macrophages, M2-polarized macrophages, CD8 cytotoxic Tcells, and Tregs using FACS. The mice in the combination treatment group that achieved long-term survival did not have a clearly defined tumor, therefore the tumor bed was harvested instead. There were no differences in the total number of 

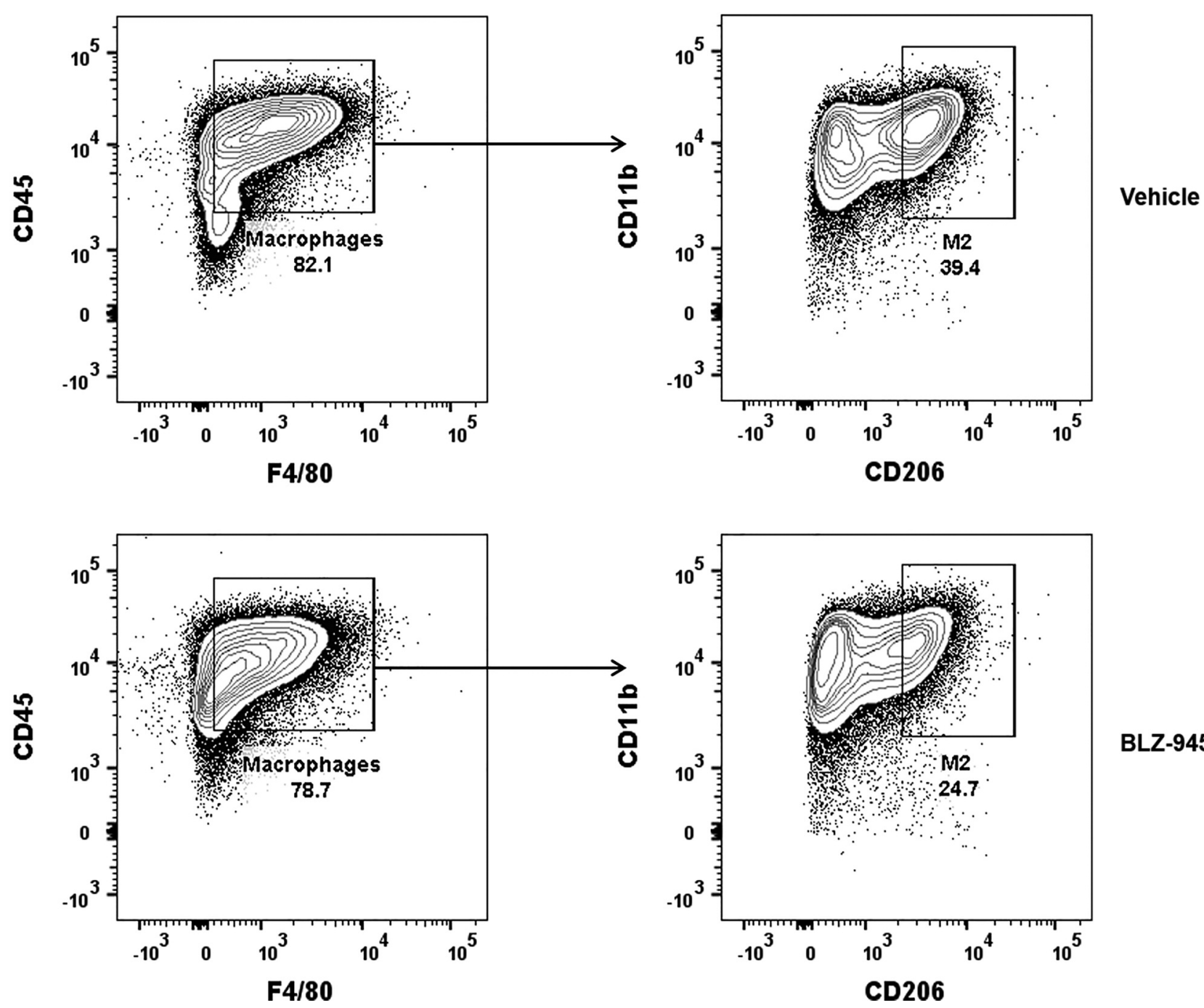

BLZ-945

Figure 1. Inhibition of colony-stimulating factor-1 receptor reduces M2 macrophage polarization. Bone marrow derived monocytes were cultured with granulocyte-macrophage colony-stimulating factor, followed by M2 induction with interleukin-4 and -13 with or without BLZ-945. Representative fluorescence-activated sorting plots are shown. Cells were initially gated by the pan-macrophage markers CD45 and F/480 to identify total macrophages. The subset of macrophages that stained for CD11b and CD206 were then selected to identify M2 macrophages. Experiments were performed in triplicates.

macrophages between any of the treatment groups (Figure 5A). However, M2 macrophage infiltration was significantly increased by RT (Figure 5B). Treatment with BLZ-945 significantly reduced M2 macrophage infiltration compared to the control arm and mitigated the RT-induced increase in these cells (Figure 5B). The number of CD8 CTLs increased with RT, although the increase did not reach statistical significance $(p=0.09)$ and BLZ-945 had minimal impact on CD8 infiltration (Figure 5C). The M2 to CD8 cell ratio was maintained at near equivalent levels in in all treatment groups, except the combination treatment group, in which it was significantly lower (Figure 5D). We observed a trend of increased Tregs in response to RT but, overall, the number of Tregs represented a relatively small population of immune cells when compared to M2 macrophages (Figure 5E). Additionally, BLZ-945 had no significant impact on Treg infiltration.

\section{Discussion}

Using an orthotopic murine model, our study shows that inhibition of CSF-1R with BLZ-945 enhanced the efficacy of RT in the treatment GBM and resulted in significantly improved survival compared to RT alone. Notably, $>70 \%$ of mice achieved long-term survival. Mechanistically, the 
A Control

BLZ-945

RT

RT+BLZ-945
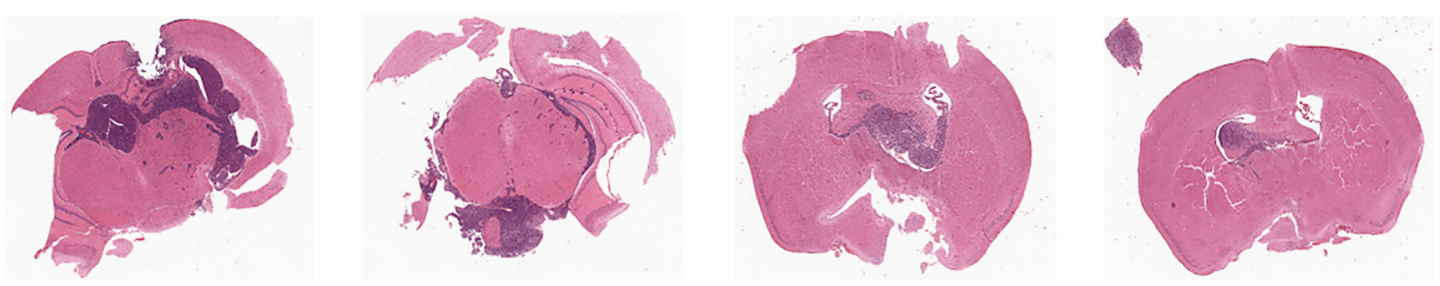

B

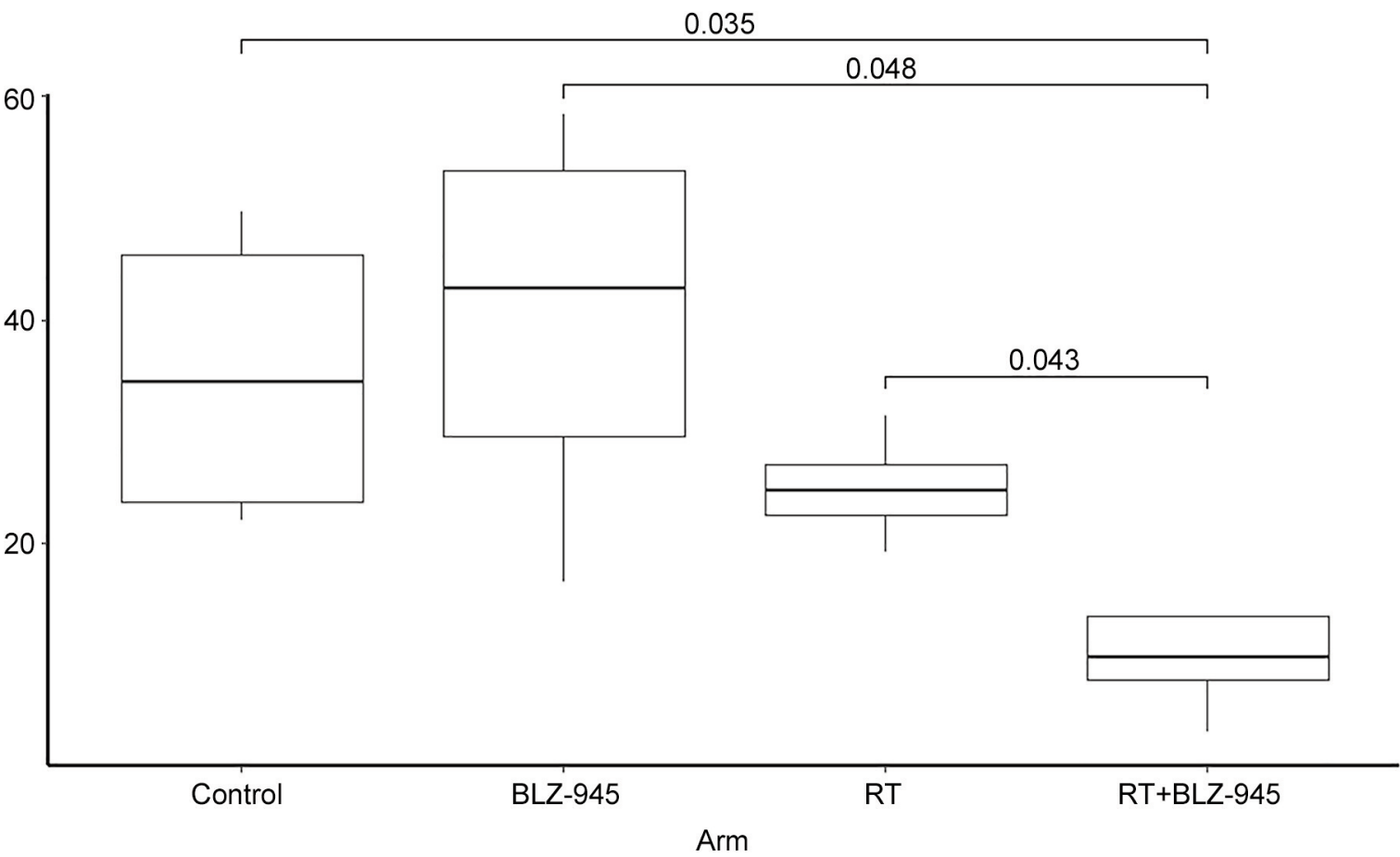

Figure 2. Inhibition of colony-stimulating factor-1 receptor potentiates the impact of radiotherapy (RT) on tumor volume. TRP murine glioblastoma cells were stereotactically injected into the right hemisphere of 6- to 8-week-old mice. On day 14, treatment with vehicle, BLZ-945 (200 mg/kg), RT (6 Gy $\times 3$ fractions), or BLZ-945 + RT was initiated, followed by daily treatment with vehicle or BLZ-945 for 2 weeks. A: Representative hematoxylin and eosin $(H \& E)$ images from each treatment group. Pink-stained tissue shows normal brain. Blue-stained tissue shows tumor cells. B: Tumor burden expressed as a percentage of normal brain volume based on $H \& E$ staining. Brains were harvested on day 10 post-treatment initiation and sectioned every $10 \mu \mathrm{M}$ through the tumor and adjacent brain tissue. Slides were imaged and segmented. An iterative learning algorithm was employed to identify normal brain tissue and tumor cells. p-Values for significant differences are shown.

enhanced efficacy appears to be related to mitigation of the increase in M2 polarized TAM infiltration, which occurs in response to RT. To the best of our knowledge, this is the first study to examine the combination of CSF-1R inhibition and RT in an immunocompetent GBM mouse model. Our findings suggest antitumor RT-mediated immunity may be potentially augmented by BLZ-945, whose safety and efficacy are currently being investigated in the treatment of multiple advanced solid tumors in a phase I/II trial (17).

Our findings are supported by other pre-clinical studies that observed a similar relationship between RT and M2 induction in several tumor models as reviewed in (14). Using a GBM model in athymic mice, one study found a significant and sustained increase in the influx of M2 TAMs into the tumor microenvironment starting 7 days following RT, and inhibition of CSF-1R by the small molecule PLX3397 suppressed this increase and resulted in improved survival (20). The improvement in survival was attributed to a decrease in microvessel density in tumors as a result of reducing the number of M2 TAMs, which are pro-angiogenic (29). However, it was not possible to make any conclusions about involvement of the adaptive immune system because of the utilization of 
A
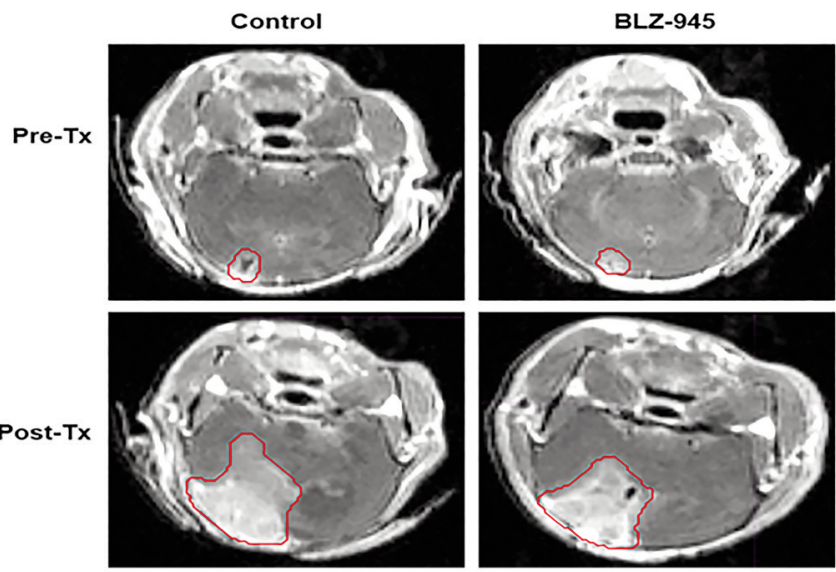
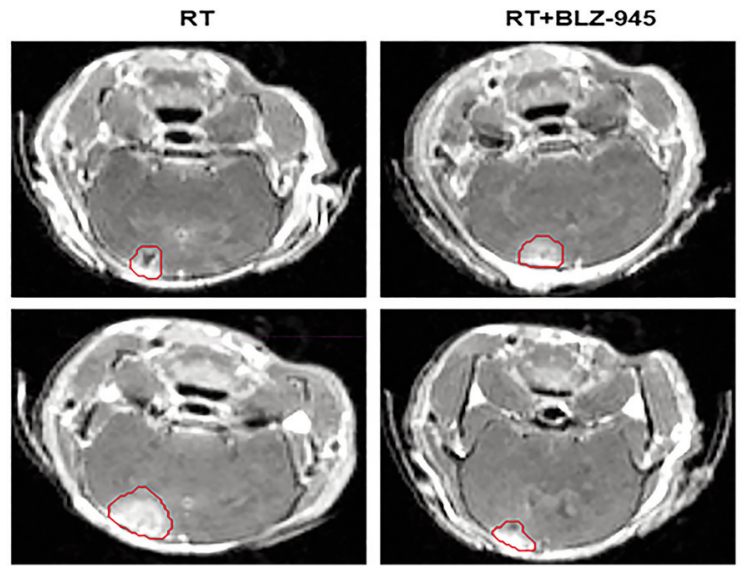

B

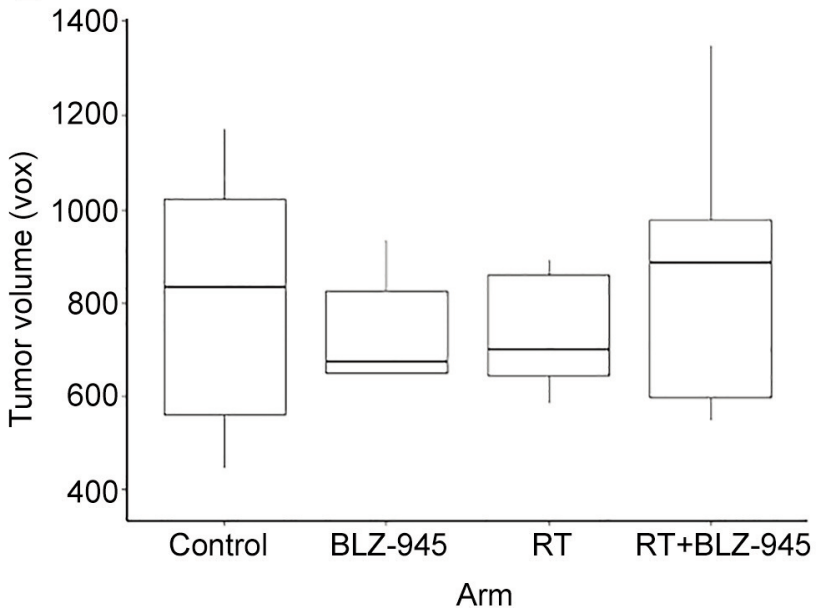

C

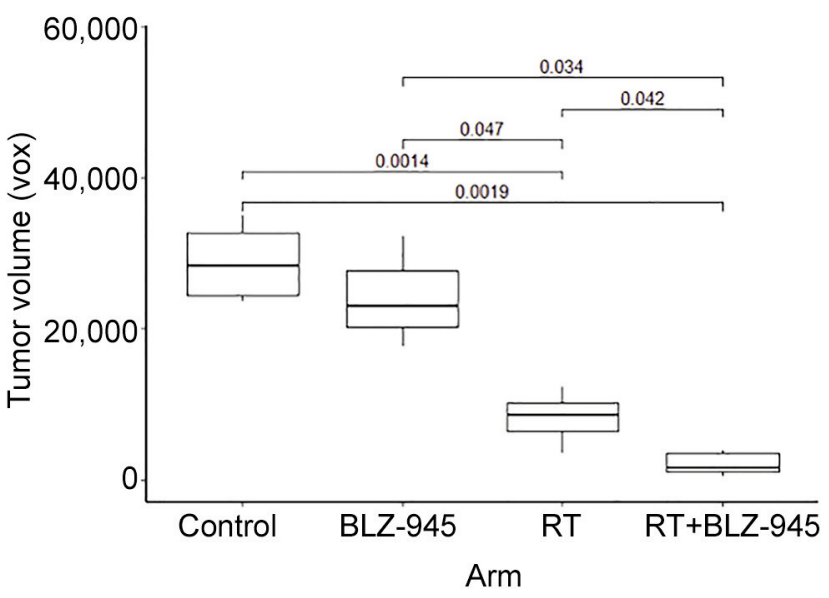

Figure 3. Inhibition of colony-stimulating factor-1 receptor potentiates radiotherapy (RT) and improves survival. TRP murine glioblastoma cells were stereotactically injected into the right hemisphere of 6- to 8-week-old mice. On day 14, treatment with vehicle, BLZ-945 (200 mg/kg), RT (6 Gy $\times 3$ fractions), or BLZ-945 with RT was initiated, followed by daily treatment with vehicle or BLZ-945 for 3 weeks. Mice were sacrificed if they became symptomatic, lost $\geq 20 \%$ weight, or reached 14 weeks. B: Sample magnetic resonance imaging of tumors prior to, and 2 weeks post-treatment (Tx). Tumors were contoured on the T1 sequence and volumes were estimated by the number of voxels. C: Boxplot of tumor volumes in each treatment group before treatment initiation. D: Boxplot of tumor volumes in each treatment group 2 weeks after initiation of treatment. p-Values for significant differences are shown.

athymic nude mice, which lack T-cells. Interestingly, in our model, eight out of eleven mice achieved long-term survival. On the other hand, despite significantly longer survival with CSF-1R inhibition compared to RT alone, none of the mice achieved long-term survival in the nude mouse model study, which used a similar experimental timeframe (20). Although not specifically tested in our studies, this discrepancy may be explained by the presence of an adaptive immune response in our model and suggests that inhibition of M2 macrophages by BLZ-945 led to long-lasting antitumor immunity.

We did not observe a significant change in survival with BLZ-945 alone, a result consistent with the above discussed study (20). This finding is further corroborated by a recently published phase II trial that treated 37 patients with the CSF1R inhibitor PLX3397 and reported no objective responses to the drug, and progression-free survival of only $8.6 \%$ at 6 months (30). In contrast, a study that utilized a transgenic mouse line reported long-term survival in ten of eleven mice treated with BLZ-945 alone (18). The study utilized a platelet-derived growth factor-B (PDGF-B) driven glioma model in which gliomagenesis is triggered by intracranial injection of a retroviral vector. The difference in outcomes is likely explained by our utilization of a very aggressive model, in which genetically engineered cells that recapitulate 


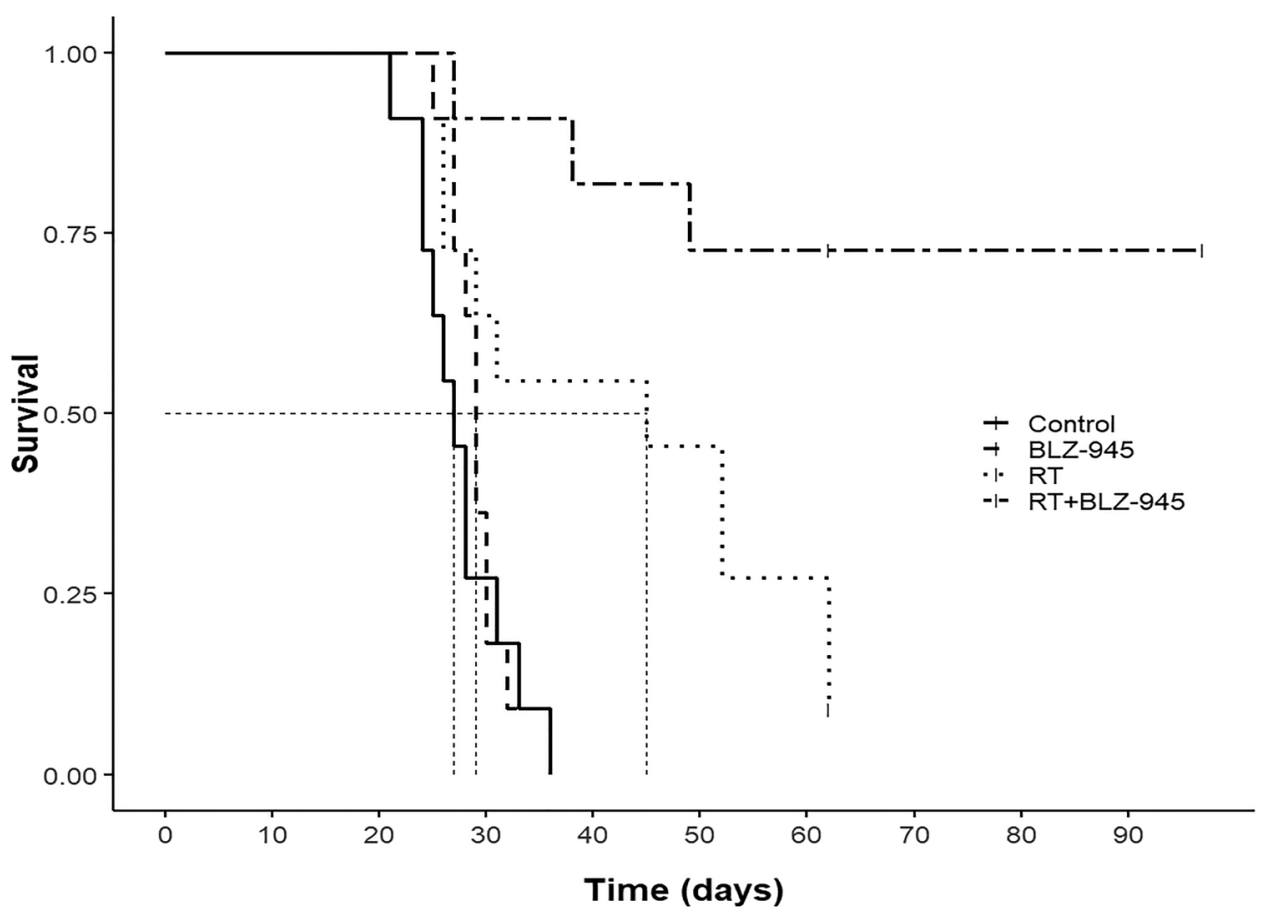

Figure 4. Kaplan-Meier estimates of survival for each treatment arm. Dashed lines denote median overall survival.

Table I. Cox regression survival analysis.

\begin{tabular}{lcccc}
\hline Treatment arm & Median OS (days) & HR & $95 \%$ CI & $p$-Value \\
\hline Control & 27 & Ref. & - & - \\
BLZ-945 & 29 & 0.74 & $0.32-1.71$ & 0.47 \\
Radiotherapy & 45 & 0.28 & $0.09-0.81$ & 0.02 \\
Radiotherapy plus BLZ-945 & Not reached & 0.05 & $0.01-0.21$ & $<0.001$ \\
\hline
\end{tabular}

HR: Hazard ratio; OS: overall survival; CI: confidence interval.

human GBM characteristics were injected intracranially, compared to PDGF-B-driven tumorigenesis.

Our results did not reveal a large percentage of $\mathrm{CD}^{+} \mathrm{CTLS}$ within the tumor microenvironment, consistent with findings from human studies (31). However, $\mathrm{CD}^{+}$cell infiltration increased in response to RT, reaching $\sim 6 \%$ of all immune cells. These results support the growing notion that the brain is not an immune-privileged organ and adaptive immunity plays an important role in GBM. Clinical studies show increased T-cell infiltration within the tumor microenvironment and brain parenchyma (32). Furthermore, higher infiltration of effector Tcells, like $\mathrm{CD}^{+} \mathrm{CTLs}$, even if in small percentages, correlates with improved patient survival (33). Unfortunately, GBM cells can usurp multiple physiological mechanisms of inducing immune tolerance to counteract the impact of increased CTL infiltration and escape immune surveillance. Chief amongst these mechanisms is M2 macrophage recruitment, which can suppress the cytotoxic activity of T-cells through production of anti-inflammatory cytokines and reduce antigen presentation by tumor cells though scavenging of immunogenic surface markers (34). Additionally, TAMs can induce a state of anergy, in which T-cells, even if abundant, are unable to attack tumor cells and may in fact become pro-tumorigenic (35). The fact that CSF1R inhibition reduces M2 TAM infiltration and the M2 to CD8 cell ratio likely reduces CD8 anergy and improves their cytotoxic activity against tumor cells.

We found a very small percentage of Tregs within the tumor microenvironment. These cells comprised $<2 \%$ of all collected immune cells. In multiple solid tumors, Tregs play a crucial part in promoting immunosuppression of CTLs and their 

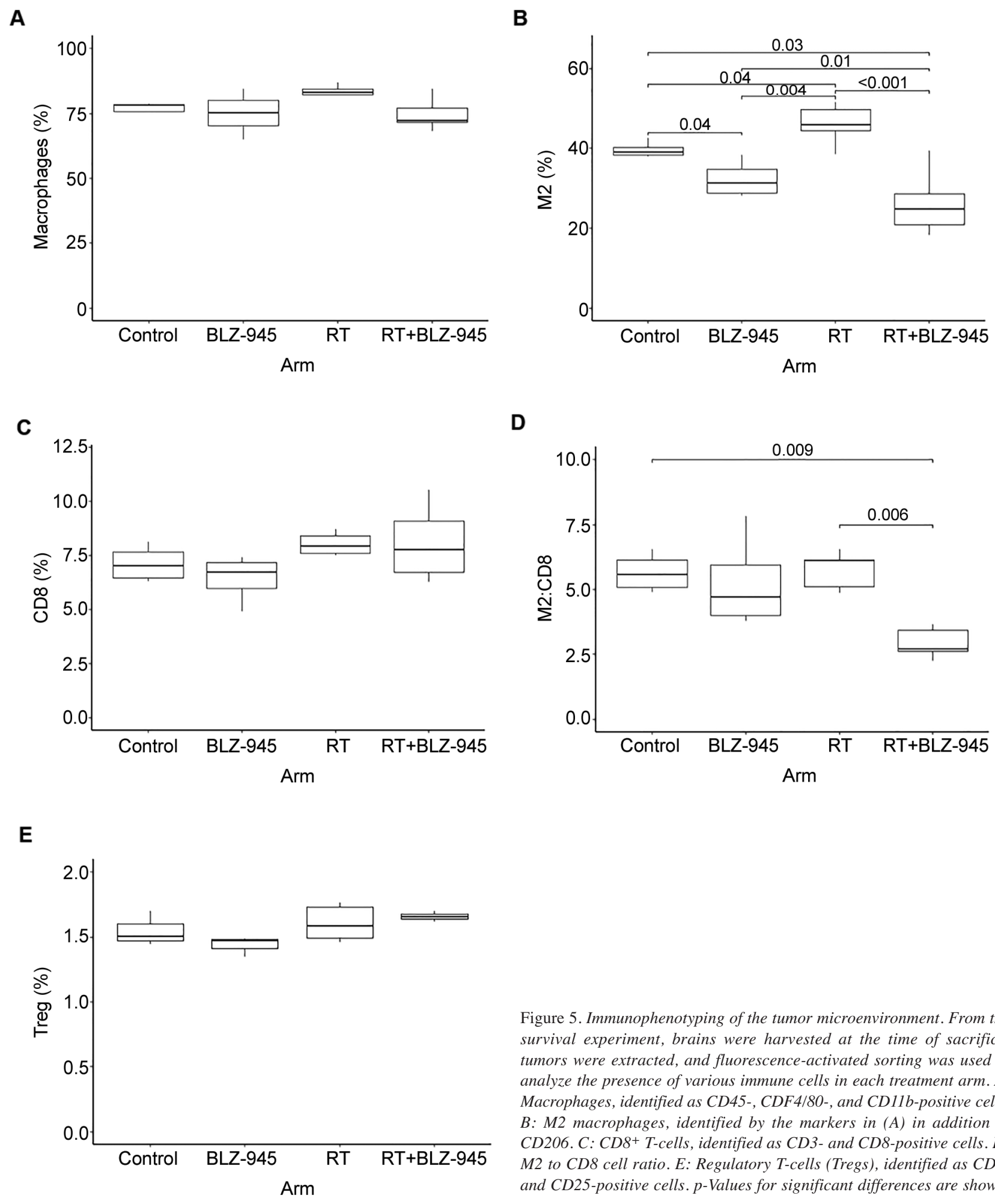

Figure 5. Immunophenotyping of the tumor microenvironment. From the survival experiment, brains were harvested at the time of sacrifice, tumors were extracted, and fluorescence-activated sorting was used to analyze the presence of various immune cells in each treatment arm. A: Macrophages, identified as CD45-, CDF4/80-, and CD11b-positive cells. $B:$ M2 macrophages, identified by the markers in (A) in addition to CD206. C: $C D 8^{+} T$-cells, identified as CD3- and CD8-positive cells. D: M2 to CD8 cell ratio. E: Regulatory T-cells (Tregs), identified as CD4and CD25-positive cells. p-Values for significant differences are shown.

recruitment into the tumor microenvironment is substantially increased in response to RT, resulting in inhibition or RTinduced antitumor immunity $(36,37)$. Additionally, Tregs also

appear to be radioresistant relative to other immune cells (37). Our findings, however, suggest that Tregs play a minor role in immune suppression by GBM and it is not surprising that 
immune checkpoint inhibitors targeting the Treg-mediated immune suppression mechanisms like PD1 and PD-L1 have shown little efficacy against GBM in recent clinical trials (7, 8). A study that analyzed primary and secondary GBM tumors from 65 patients identified Tregs in only $\sim 40 \%$ of patients and these cells represented $<1 \%$ of all T-cells (33).

We did not find a significant reduction in the overall number of macrophages within the tumor microenvironment and brain parenchyma with inhibition of CSF-1R. Rather, the reduction in M2 macrophages was likely the result of inhibiting M2 polarization as illustrated by the results of the in vitro assays, in which induction of M2 polarization by the cytokines IL14 and IL13 was significantly diminished in the presence of BLZ-945. These findings are corroborated by the aforementioned study, which used a PDGF-B derived gliomagenesis model (18). The authors found inhibition of CSF-1R reduced macrophage proliferation in vitro but had no impact on the number of TAMs in vivo. However, the study found a significant reduction in M2 TAMs with CSF-1 inhibition. The discrepancy between the in vivo and in vitro results was explained by additional experiments, which found tumor cells produced cytokines that protected macrophages against apoptosis in the absence of CSF$1 \mathrm{R}$ signaling (18). Microarray expression profiling revealed down-regulation of M2 macrophage-specific genes with CSF$1 \mathrm{R}$ inhibition. The authors did not study the interaction between RT and M2 TAMs.

Mono-immunotherapy has thus far shown little efficacy in the treatment of GBM, but the pre-clinical and clinical evidence summarized here suggests that the combination of RT and immunotherapy, especially anti-M2 macrophage therapy, has significant therapeutic potential. Similarly, while indoleamine 2,3-dioxygenase inhibitors are able to modulate immune surveillance of tumor cells, studies have found them insufficient to lead to significant therapeutic benefit as a monotherapy, but they do have a synergistic therapeutic effect when combined with RT $(5,38)$.

In conclusion, our results show inhibition of CSF-1R with BLZ-945 enhanced the efficacy of RT in the treatment of GBM in a pre-clinical model. Correlative studies suggest BLZ-945 improves response to RT by reducing M2 polarization within the tumor microenvironment and likely creating a more pro-inflammatory milieu. Combination of RT and BLZ-945 represents a promising therapeutic strategy to be investigated clinically in GBM.

\section{Conflicts of Interest}

The Authors have no conflicts of interest to declare

\section{Authors' Contributions}

Muayad F. Almahariq: contributed substantially to the study's conception and design, performing experiments, data acquisition and analysis, and writing of the article. Thomas J. Quinn: contributed substantially to the study's conception and design, performing experiments, data acquisition and analysis, and writing of the manuscript. Pravin Kesarwani: contributed substantially to performing experiments and data acquisition and analysis. Shiva Kant: contributed substantially to performing experiments and data acquisition and analysis. C. Ryan Miller: contributed substantially to data interpretation and revision of the article. Prakash Chinnaiyan: contributed substantially to the study's conception and design, data interpretation, and revision of the article.

\section{Acknowledgements}

This work was supported by the National Institute of Health (NIH)/National Institute of Neurological Disorders and Stroke (NINDS) (R01 NS110838, R21NS090087), American Cancer Society (RSG-11-029-01), Bankhead-Coley Cancer Research Program and Cancer Research, and Seed Grant Awards from Beaumont Health to Prakash Chinnaiyan.

\section{References}

1 Ostrom QT, Gittleman H, Fulop J, Liu M, Blanda R, Kromer C, Wolinsky Y, Kruchko C and Barnholtz-Sloan JS: CBTRUS statistical report: Primary brain and central nervous system tumors diagnosed in the United States In 2008-2012. Neuro Oncol 17(Suppl 4): iv1-iv62, 2015. PMID: 26511214. DOI: 10.1093/neuonc/nov189

2 Stupp R, Hegi ME, Mason WP, van den Bent MJ, Taphoorn MJ, Janzer RC, Ludwin SK, Allgeier A, Fisher B, Belanger K, Hau P, Brandes AA, Gijtenbeek J, Marosi C, Vecht CJ, Mokhtari K, Wesseling P, Villa S, Eisenhauer E, Gorlia T, Weller M, Lacombe D, Cairncross JG, Mirimanoff RO on behalf of the European Organisation for Research and Treatment of Cancer Brain Tumour and Radiation Oncology Groups the National Cancer Institute of Canada Clinical Trials Group: Effects of radiotherapy with concomitant and adjuvant temozolomide versus radiotherapy alone on survival in glioblastoma in a randomised phase III study: 5-Year analysis of the EORTCNCIC trial. Lancet Oncol 10(5): 459-466, 2009. PMID: 19269895. DOI: 10.1016/S1470-2045(09)70025-7

3 Jackson CM, Choi J and Lim M: Mechanisms of immunotherapy resistance: Lessons from glioblastoma. Nat Immunol 20(9): 1100-1109, 2019. PMID: 31358997. DOI: 10.1038/s41590-0190433-y

4 Brocard E, Oizel K, Lalier L, Pecqueur C, Paris F, Vallette FM and Oliver L: Radiation-induced PGE2 sustains human glioma cells growth and survival through EGF signaling. Oncotarget 6(9): 68406849, 2015. PMID: 25749386. DOI: 10.18632/oncotarget.3160

5 Kesarwani P, Prabhu A, Kant S, Kumar P, Graham SF, Buelow KL, Wilson GD, Miller CR and Chinnaiyan P: Tryptophan metabolism contributes to radiation-induced immune checkpoint reactivation in glioblastoma. Clin Cancer Res 24(15): 36323643, 2018. PMID: 29691296. DOI: 10.1158/1078-0432.CCR18-0041

6 Tabatabaei P, Visse E, Bergstrom P, Brannstrom T, Siesjo P and Bergenheim AT: Radiotherapy induces an immediate inflammatory reaction in malignant glioma: A clinical microdialysis study. J Neurooncol 131(1): 83-92, 2017. PMID: 27664151. DOI: $10.1007 / \mathrm{s} 11060-016-2271-1$ 
7 Namouni F: Primary Endpoint Not Met in Phase III CheckMate498 Trial of MGMT-Unmethylated GBM. Available at: https://www.targetedonc.com/news/primary-endpoint-notmet-in-phase-iii-checkmate498-trial-of-mgmtunmethylated-gbm [Last accessed October 22, 2020]

8 Reardon DA, Omuro A, Brandes AA, Rieger J, Wick A, Sepulveda J, Phuphanich S, de Souza P, Ahluwalia MS, Lim M, Vlahovic G and Sampson J: Os10.3 randomized phase 3 study evaluating the efficacy and safety of nivolumab $v s$. bevacizumab in patients with recurrent glioblastoma: Checkmate 143. NeuroOncology 19(suppl_3): iii21, 2017. PMID: 32437507. DOI: 10.1001/jamaoncol.2020.1024

9 Caccese M, Indraccolo S, Zagonel V and Lombardi G: Pd-1/pd11 immune-checkpoint inhibitors in glioblastoma: A concise review. Crit Rev Oncol Hematol 135:128-134, 2019. PMID: 30819441. DOI: 10.1016/j.critrevonc.2018.12.002

10 Wang Q, Hu B, Hu X, Kim H, Squatrito M, Scarpace L, deCarvalho AC, Lyu S, Li P, Li Y, Barthel F, Cho HJ, Lin YH, Satani N, Martinez-Ledesma E, Zheng S, Chang E, Sauve CG, Olar A, Lan ZD, Finocchiaro G, Phillips JJ, Berger MS, Gabrusiewicz KR, Wang G, Eskilsson E, Hu J, Mikkelsen T, DePinho RA, Muller F, Heimberger AB, Sulman EP, Nam DH and Verhaak RGW: Tumor evolution of glioma - intrinsic gene expression subtypes associates with immunological changes in the microenvironment. Cancer Cell 32(1): 42-56.e46, 2017. PMID: 28697342. DOI: 10.1016/j.ccell.2017.06.003

11 Wei SC, Duffy CR and Allison JP: Fundamental mechanisms of immune checkpoint blockade therapy. Cancer Discov 8(9): 10691086, 2018. PMID: 30115704. DOI: 10.1158/2159-8290.CD-180367

12 Hussain SF, Yang D, Suki D, Aldape K, Grimm E and Heimberger $\mathrm{AB}$ : The role of human glioma-infiltrating microglia/macrophages in mediating antitumor immune responses. Neuro Oncol 8(3): 261279, 2006. PMID: 16775224. DOI: 10.1215/15228517-2006-008

13 Murray PJ: Macrophage polarization. Annu Rev Physiol 79: 541-566, 2017. PMID: 27813830. DOI: 10.1146/annurevphysiol-022516-034339

14 Vatner RE and Formenti SC: Myeloid-derived cells in tumors: Effects of radiation. Semin Radiat Oncol 25(1): 18-27, 2015. PMID: 25481262. DOI: 10.1016/j.semradonc.2014.07.008

15 Poh AR and Ernst M: Targeting macrophages in cancer: From bench to bedside. Front Oncol 8: 49, 2018. PMID: 29594035. DOI: $10.3389 /$ fonc 2018.00049

16 Jeannin P, Paolini L, Adam C and Delneste Y: The roles of CSFs on the functional polarization of tumor-associated macrophages. FEBS 285(4): 680-699, 2018. PMID: 29171156. DOI: $10.1111 /$ febs. 14343

17 Phase I/II study of BLZ945 single agent or BLZ945 in combination with PDR001 in advanced solid tumors. ClinicalTrials.gov, 2019. Available at: https://clinicaltrials.gov/ct2/show/NCT02829723

18 Pyonteck SM, Akkari L, Schuhmacher AJ, Bowman RL, Sevenich L, Quail DF, Olson OC, Quick ML, Huse JT, Teijeiro V, Setty M, Leslie CS, Oei Y, Pedraza A, Zhang J, Brennan CW, Sutton JC, Holland EC, Daniel D and Joyce JA: CSF-1R inhibition alters macrophage polarization and blocks glioma progression. Nat Med 19(10): 1264-1272, 2013. PMID: 24056773. DOI: $10.1038 / \mathrm{nm} .3337$

19 Quail DF, Bowman RL, Akkari L, Quick ML, Schuhmacher AJ, Huse JT, Holland EC, Sutton JC and Joyce JA: The tumor microenvironment underlies acquired resistance to CSF-1R inhibition in gliomas. Science 352(6288): aad3018, 2016. PMID: 27199435. DOI: $10.1126 /$ science.aad3018

20 Stafford JH, Hirai T, Deng L, Chernikova SB, Urata K, West BL and Brown JM: Colony stimulating factor 1 receptor inhibition delays recurrence of glioblastoma after radiation by altering myeloid cell recruitment and polarization. Neuro Oncol 18(6): 797-806, 2016. PMID: 26538619. DOI: 10.1093/neuonc/nov272

21 Kesarwani P, Prabhu A, Kant S and Chinnaiyan P: Metabolic remodeling contributes towards an immune-suppressive phenotype in glioblastoma. Cancer Immunol Immunother 68(7): 1107-1120, 2019. PMID: 31119318. DOI: 10.1007/s00262-01902347-3

22 Roszer T: Understanding the mysterious M2 macrophage through activation markers and effector mechanisms. Mediators Inflamm 2015: 816460, 2015. PMID: 26089604. DOI: $10.1155 / 2015 / 816460$

23 Vitucci M, Karpinich NO, Bash RE, Werneke AM, Schmid RS, White KK, McNeill RS, Huff B, Wang S, Van Dyke T and Miller CR: Cooperativity between MAPK and PI3K signaling activation is required for glioblastoma pathogenesis. Neuro Oncol 15(10): 1317-1329, 2013. PMID: 23814263. DOI: 10.1093/neuonc/not084

24 Schmid RS, Simon JM, Vitucci M, McNeill RS, Bash RE, Werneke AM, Huey L, White KK, Ewend MG, Wu J and Miller $\mathrm{CR}$ : Core pathway mutations induce de-differentiation of murine astrocytes into glioblastoma stem cells that are sensitive to radiation but resistant to temozolomide. Neuro Oncol 18(7): 962-973, 2016. PMID: 26826202. DOI: 10.1093/neuonc/nov321

25 McNeill RS, Canoutas DA, Stuhlmiller TJ, Dhruv HD, Irvin DM, Bash RE, Angus SP, Herring LE, Simon JM, Skinner KR, Limas JC, Chen X, Schmid RS, Siegel MB, Van Swearingen AED, Hadler MJ, Sulman EP, Sarkaria JN, Anders CK, Graves LM, Berens ME, Johnson GL and Miller CR: Combination therapy with potent PI3K and MAPK inhibitors overcomes adaptive kinome resistance to single agents in preclinical models of glioblastoma. Neuro Oncol 19(11): 1469-1480, 2017. PMID: 28379424. DOI: $10.1093 /$ neuonc/nox044

26 Wilson TG, Hanna A, Recknagel J, Pruetz BL, Baschnagel AM and Wilson GD: Prognostic significance of mtor expression in HPV positive and negative head and neck cancers treated by chemoradiation. Head Neck, 2019. PMID: 31657099. DOI: 10.1002/hed.25983

27 Setrerrahmane $\mathrm{S}$ and $\mathrm{Xu} \mathrm{H}$ : Tumor-related interleukins: Old validated targets for new anti-cancer drug development. Mol Cancer 16(1): 153, 2017. PMID: 28927416. DOI: 10.1186/s12943-017$0721-9$

28 El Meskini R, Iacovelli AJ, Kulaga A, Gumprecht M, Martin PL, Baran M, Householder DB, Van Dyke T and Weaver Ohler Z: A preclinical orthotopic model for glioblastoma recapitulates key features of human tumors and demonstrates sensitivity to a combination of MEK and PI3K pathway inhibitors. Dis Model Mech 8(1): 45-56, 2015. PMID: 25431423. DOI: 10.1242/dmm. 018168

29 Mantovani A, Biswas SK, Galdiero MR, Sica A and Locati M: Macrophage plasticity and polarization in tissue repair and remodelling. J Pathol 229(2): 176-185, 2013. PMID: 23096265. DOI: $10.1002 /$ path. 4133

30 Butowski N, Colman H, De Groot JF, Omuro AM, Nayak L, Wen PY, Cloughesy TF, Marimuthu A, Haidar S, Perry A, Huse J, Phillips J, West BL, Nolop KB, Hsu HH, Ligon KL, Molinaro 
AM and Prados M: Orally administered colony stimulating factor 1 receptor inhibitor PLX3397 in recurrent glioblastoma: An Ivy Foundation Early Phase Clinical Trials Consortium phase II study. Neuro Oncol 18(4): 557-564, 2016. PMID: 26449250. DOI: $10.1093 /$ neuonc/nov245

31 Orrego E, Castaneda CA, Castillo M, Bernabe LA, Casavilca S, Chakravarti A, Meng W, Garcia-Corrochano P, Villa-Robles MR, Zevallos R, Mejia O, Deza P, Belmar-Lopez C and Ojeda L: Distribution of tumor-infiltrating immune cells in glioblastoma. CNS Oncol 7(4): CNS21, 2018. PMID: 30299157. DOI: 10.2217/cns-2017-0037

32 Brown NF, Carter TJ, Ottaviani D and Mulholland P: Harnessing the immune system in glioblastoma. Br J Cancer 119(10): 11711181, 2018. PMID: 30393372. DOI: 10.1038/s41416-018-0258-8

33 Lohr J, Ratliff T, Huppertz A, Ge Y, Dictus C, Ahmadi R, Grau S, Hiraoka N, Eckstein V, Ecker RC, Korff T, von Deimling A, Unterberg A, Beckhove P and Herold-Mende C: Effector T-cell infiltration positively impacts survival of glioblastoma patients and is impaired by tumor-derived TGF-beta. Clin Cancer Res 17(13): 4296-4308, 2011. PMID: 21478334. DOI: 10.1158/10780432.CCR-10-2557

34 Genard G, Lucas S and Michiels C: Reprogramming of tumorassociated macrophages with anticancer therapies: Radiotherapy versus chemo- and immunotherapies. Front Immunol 8: 828, 2017. PMID: 28769933. DOI: 10.3389/fimmu.2017.00828

35 Seifert L, Werba G, Tiwari S, Ly NNG, Nguy S, Alothman S, Alqunaibit D, Avanzi A, Daley D, Barilla R, Tippens D, TorresHernandez A, Hundeyin M, Mani VR, Hajdu C, Pellicciotta I, Oh P, Du K and Miller G: Radiation therapy induces macrophages to suppress immune responses against pancreatic tumors in mice. Gastroenterology 150(7): 1659-1672 e1655, 2016. PMID: 26946344. DOI: 10.1053/j.gastro.2016.02.070
36 Kachikwu EL, Iwamoto KS, Liao YP, DeMarco JJ, Agazaryan N, Economou JS, McBride WH and Schaue D: Radiation enhances regulatory T-cell representation. Int J Radiat Oncol Biol Phys 81(4): 1128-1135, 2011. PMID: 21093169. DOI: 10.1016/j.ijrobp.2010.09.034

37 Liu S, Sun X, Luo J, Zhu H, Yang X, Guo Q and Song Y: Effects of radiation on T-regulatory cells in normal states and cancer: Mechanisms and clinical implications. Am J Cancer Res 5(11): 3276-3285, 2015. PMID: 26807310.

38 Ladomersky E, Zhai L, Lenzen A, Lauing KL, Qian J, Scholtens DM, Gritsina G, Sun X, Liu Y, Yu F, Gong W, Jiang B, Tang T, Patel R, Platanias LC, James CD, Stupp R, Lukas RV, Binder DC and Wainwright DA: IDO1 inhibition synergizes with radiation and PD-1 blockade to durably increase survival against advanced glioblastoma. Clin Cancer Res 24(11): 2559-2573, 2018. PMID: 29500275. DOI: 10.1158/1078-0432.CCR-17-3573

Received September 10, 2020

Revised October 17, 2020

Accepted October 22, 2020 